Rev Inv Vet Perú 2005; 16 (1):90-95

\title{
EVALUACIÓN DEL pH EN CANALES DE TOROS HOLSTEIN (Bos taurus) Y NELORE (Bos indicus)
}

\author{
Gino Mariño A. ${ }^{1}$, Miguel Vilca L. ${ }^{2}$ y Daphne Ramos D. ${ }^{2}$
}

\section{Abstract}

With the objective to find differences racial, the curve of variation of $\mathrm{pH}$ of the meat in 50 bulls Holstein (Bos taurus) and 50 bulls Nelore (Bos indicus) was determined, with ages between 12-24 months The $\mathrm{pH}$ measurements was taken in the Longissimus dorsi muscle after 1, 2, 3, 4, 5, 6, 8, 10, 12, 15, 18, 24 hours post mortem. Data was submitted to regression analysis. The $\mathrm{pH}$ values were expressed through a cubic regression equation, (in Holstein cattle $\mathrm{w}=7.107273-0.314504 \mathrm{x}+0.019409 \mathrm{x}^{2}-0.000374 \mathrm{x}^{3}$ with $R^{2}=0.814$ and in Nelore was $w=7.005286-0.309965 x+0.019198 x^{2}-0.00378 x^{3}$ with $\left.\mathrm{R}^{2}=0.767\right)$. those of the Holstein race were slightly greater to those of the Nelore race, presenting racial differences $(\mathrm{p}<00.5)$, however in both races they follow similar tendency during the first $24 \mathrm{~h}$ post mortem and reach an suitable degree of acidity to inhibit the microbial growth and to promote a good meat quality.

Key words: $\mathrm{pH}$, longissimus dorsi, Holstein, Nelore, beef

\section{RESUMEN}

Con el objetivo de encontrar diferencias raciales, se determinó la curva de variación del pH de la carne en 50 toros Holstein (Bos taurus) y 50 toros Nelore (Bos indicus), con edades que fluctuaban entre 12 a 24 meses. El pH se midió en el músculo Longissimus dorsi después de 1, 2, 3, 4, 5, 6, 8, 10, 12, 15, 18, 24 horas del sacrificio. Los valores de $\mathrm{pH}$ obtenidos definieron una ecuación de regresión tipo cúbica (en bovinos Holstein fue $\mathrm{w}=7.107273-0.314504 \mathrm{x}+0.019409 \mathrm{x}^{2}-0.000374 \mathrm{x}^{3}$ con $\mathrm{R}^{2}=0.814 \mathrm{y}$ en Nelore fue $\mathrm{w}=7.005286-0.309965 \mathrm{x}+0.019198 \mathrm{x}^{2}-0.00378 \mathrm{x}^{3}$ con $\mathrm{R}^{2}=0.767$ ). Los valores de $\mathrm{pH}$ en la carne mostraron un rápido descenso durante las 12 primeras horas. Los valores de $\mathrm{pH}$ de la raza Holstein fueron ligeramente mayores a los del Nelore $(\mathrm{p}<0.05)$, aunque en ambas razas siguen similar tendencia durante las primeras 24 horas post mortem y alcanzan un grado de acidez adecuado para inhibir el crecimiento microbiano y promover una buena calidad cárnica.

Palabras clave: $\mathrm{pH}$, longissimus dorsi, Holstein, Nelore, carne

\footnotetext{
${ }^{1}$ Práctica privada

${ }^{2}$ Laboratorio de Salud Pública y Salud Ambiental, FMV-UNMSM
} 


\section{INTRODUCCION}

Los procesos fisiológicos y bioquímicos que ocurren en el organismo del animal, luego del sacrificio, están directamente relacionados con el rápido descenso de la cantidad de oxígeno presente en el torrente sanguineo. Los procesos post mortem, propiamente dichos, comienzan en la carne luego de la muerte biológica de los músculos (Fehlhaber y Janetschke, 1995). Los músculos ya no pueden obtener energía a través de la respiración (vía aeróbica), y prosiguen sin él (vía anaeróbica) (Murray et al., 2001). Esta energía está marcada por el proceso de degradación y resintesis de ATP. Se produce ácido láctico que no puede ser metabolizado ni transformado (Pränld et al., 1994). Entonces el ácido láctico se acumula en el músculo en una cantidad que depende de las reservas de glucógeno, hasta que su producción se interrumpe, bien sea por el agotamiento del glucógeno, o porque el descenso del $\mathrm{pH}$ alcanza valores que inhiben las reacciones enzimáticas (Sánchez, 1999).

La velocidad del descenso del $\mathrm{pH}$ después de la muerte del animal constituye uno de los factores cruciales de la transformación del músculo en carne, así como en la definición de la calidad futura de los productos preparados a partir de ella (Pardi et al, 1993). El valor $\mathrm{pH}$ del músculo a las 24 horas post mortem es otro factor que influye sobre aspectos de la calidad de la carne, como por ejemplo de su capacidad de retención de agua, así como las propiedades organolépticas de aroma, sabor, terneza suculencia y color (Devine et al., 1983), así como la inhibición del crecimiento microbiano (Sánchez, 1999).

Existen diferencias con respecto al ganado Bos indicus, el cual criado bajo un sistema extensivo, puede manifestar nerviosismo y comportamiento arisco o bravio (Alves. 1980). En cambio, el Holstein (Bos taurus) que proviene de hatos lecheros estabulados es criado bajo un sistema intensivo, además de pasar un periodo intensivo de engorde estabulado (Flores, 1997). Shackelford et al. en 1995, demostraron las diferencias genéticas raciales en los músculos, evaluando, entre otras caracteristicas, la ternura de la carne.

El objetivo del presente estudio fue determinar las curvas de variación del $\mathrm{pH}$ de la carne de 2 razas de ganado bovino durante las primeras 24 horas post mortem, con el fin de emplearlas en la predicción de la duración y calidad de la carne.

\section{MLTERALES Y MF́rodos}

\section{Lugar de estudio}

El trabajo se realizó en el Camal Frigorífico Jose Olaya SAC, ubicado en el kilómetro 18.5 de la Panamericana Sur, distrito de Chorrillos, Lima, y en el Laboratorio de Salud Pública y Salud Ambiental de la Facultad de Medicina Veterinaria de la Universidad Nacional Mayor de San Marcos.

\section{Animales}

Se utilizaron 100 toros; la mitad de raza Holstein (Bos taurus) y la otra mitad de raza Nelore (Bos indicus), procedentes de centros de engorde de la zona, cuyas edades fluctuaban entre 12 a 30 meses de edad ( 2 a 4 dientes). Todas las canales fueron clasificadas por el Inspector Médico Veterinario.

\section{Procedimiento}

Se midió el $\mathrm{pH}$ y la temperatura en la porción lumbar del músculo Longissimus dorsi de cada carcasa a las $1,2,3,4,5,6,8$, $10,12,15,18$ y 24 horas post mortem. Para este efecto se hizo un corte de $1 \mathrm{~cm}$ de largo por $5 \mathrm{~cm}$ de profundidad. Se insertó el electrodo y se leyó después de 30 segundos. La lectura del pH se realizó con un potenciómetro digital portátil (Hanna, modelo Checher 3), dotado de un electrodo de inserción modelo Fc $200 \mathrm{~B}$, con resolución de 0.01 unidades de pH. La lectura de la temperatura se realizó con un termómetro portátil de inser- 
ción, digital, marca Multi-thermometer, con rango de $-50{ }^{\circ} \mathrm{C} \mathrm{a}+200^{\circ} \mathrm{C}$, con una precisión de $0.01^{\circ} \mathrm{C}$.

La temperatura se utilizó para calcular el valor actual del $\mathrm{pH}$ corregido a $25{ }^{\circ} \mathrm{C}$ (Barnett, 1999):

$$
\begin{aligned}
\mathrm{pH} \text { actual }= & \underset{\text { rrección }(\mathrm{FC})}{\mathrm{pH} \text { medido-Factor de co- }} \\
\text { donde } \mathrm{FC}= & \begin{aligned}
0.03(\text { temperatura actual - } \\
\left.25^{\circ} \mathrm{C}\right)
\end{aligned}
\end{aligned}
$$

\section{Análisis estadísticos}

Los valores de $\mathrm{pH}$ actuales se sometieron a un análisis de modelos de regresión para definir las características del modelo matemático que corresponde a la distribución de los datos obtenidos, utilizando el paquete estadístico SPSS v. 10.07. Se determinó el co- eficiente de regresión, así como el tipo de función que mejor se ajusta a las observaciones de $\mathrm{pH}$ cárnico:

$$
i=\beta_{0}-\beta_{1} x+\beta_{2} x^{2}-\beta_{3} x^{3}
$$

Donde:

$i=$ Representa el valor del $\mathrm{pH}$

$\mathrm{X}=$ Representa el tiempo de medición

$\beta_{(1,2,3)}=$ Constantes

Se estimó la curva modelo de la variación de pH para cada raza, así como los intervalos de confianza para cada valor estimado de $\mathrm{pH}$ a través del paquete estadístico SAS v. 6.4 .

Los datos estimados de $\mathrm{pH}$ se sometieron a análisis de varianza utilizando un diseño experimental en bloques.

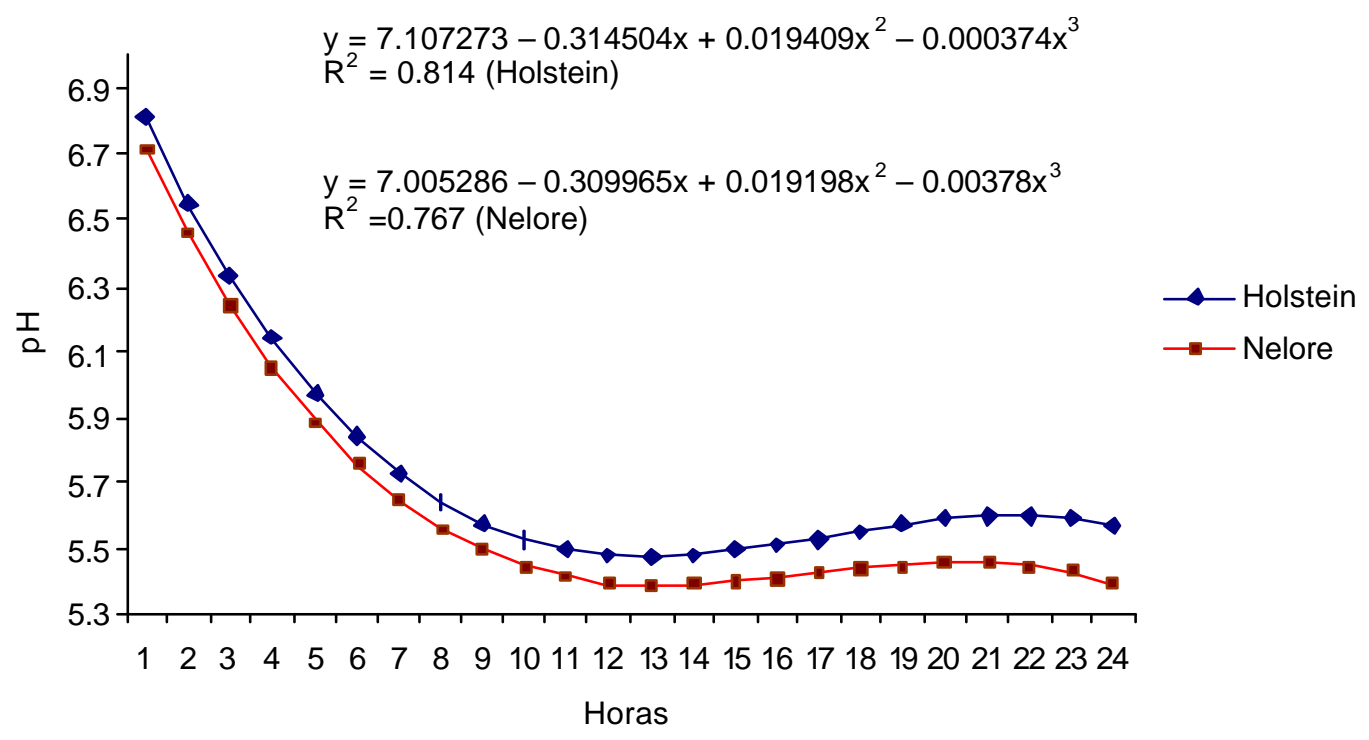

Figura 1. Curvas de variación post mortem del pH en el músculo Longissimus dorsi, en las razas Holstein (Bos taurus) y Nelore (Bos indicus) 
Cuadro 1. Valores estimados del pH cárnico obtenidos por cúbica regresión en toros de las razas Holstein y Nelore durante las primeras 24 horas post mortem

\begin{tabular}{|c|c|c|c|c|}
\hline \multirow{2}{*}{$\begin{array}{c}\text { Horas } \\
\text { post mortem }\end{array}$} & \multicolumn{2}{|c|}{ Holstein } & \multicolumn{2}{|c|}{ Nelore } \\
\hline & $\mathrm{pH}$ & $I^{\prime} C^{\prime}$ & $\mathrm{pH}$ & IC \\
\hline 1 & 6.81 & 0.00 & 6.71 & 0.00 \\
\hline 2 & 6.55 & 0.00 & 6.46 & 0.00 \\
\hline 3 & 6.33 & 0.00 & 6.24 & 0.01 \\
\hline 4 & 6.14 & 0.01 & 6.05 & 0.01 \\
\hline 5 & 5.97 & 0.01 & 5.89 & 0.01 \\
\hline 6 & 5.84 & 0.01 & 5.75 & 0.01 \\
\hline 7 & 5.73 & 0.01 & 5.65 & 0.01 \\
\hline 8 & 5.64 & 0.01 & 5.56 & 0.01 \\
\hline 9 & 5.58 & 0.01 & 5.50 & 0.02 \\
\hline 10 & 5.53 & 0.02 & 5.45 & 0.02 \\
\hline 11 & 5.50 & 0.02 & 5.42 & 0.02 \\
\hline 12 & 5,48 & 0.02 & 5.40 & 0.02 \\
\hline 13 & 5.48 & 0.02 & 5.39 & 0.02 \\
\hline 14 & 5.48 & 0.02 & 5.39 & 0.02 \\
\hline 15 & 5.49 & 0.02 & 5.40 & 0.03 \\
\hline 16 & 5.51. & 0.02 & 5.41 & 0.03 \\
\hline 17 & 5.53 & 0.03 & 5.43 & 0.03 \\
\hline 18 & 5.55 & 0.03 & 5.44 & 0.03 \\
\hline 19 & 5.57 & 0.03 & 5.45 & 0.03 \\
\hline 20 & 5.59 & 0.03 & 5.46 & 0.04 \\
\hline 21 & 5.60 & 0.03 & 5.46 & 0.04 \\
\hline 22 & 5.60 & 0.03 & 5.45 & 0.04 \\
\hline 23 & 5.59 & 0.03 & 5.43 & 0.04 \\
\hline 24 & 5.57 & 0.04 & 5.40 & 0.04 \\
\hline
\end{tabular}

${ }^{1}$ Intervalo de confianza del $95 \%$

\section{Resclatados y Discusión}

El modelo matemático de regresión que mejor se ajustó a los valores de pH durante las primeras 24 horas post mortem fue el cúbico (Fig. 1).

De acuerdo a los resultados de la regresión cúbica se obtuvieron los valores estimados de pH en el músculo Longissimus dorsi para cada raza, durante las primeras 24 horas de post mortem (Cuadro 1).

Se encontraron diferencias entre razas observándose que los valores de pH más al- tos corresponden al Holstein (Cuadro 1, Fig. $1, \mathrm{p}<0.05$ ). Estas diferencias pueden deberse a la dieta, al temperamento del animal, que en el caso del Nelore, por ser un animal más nervioso, consume glucógeno muscular (transformándolo en ácido láctico) más rápido que el Holstein, cuyo temperamento es más calmado.

Los valores estimados de $\mathrm{pH}$ de la carne a las 24 horas post mortem fueron de 5.57 para los animales Holstein y de 5.40 para los Nelore. Estos valores son lo suficientemente ácidos para inhibir el crecimiento microbiano (Sánchez, 1999); y cuando los valores de pH 
comienzan a subir y llegan a 6.2 - 6.5, aparece el peligro de una alteración de origen bacteriológico (Effenberger y Shotte, 1972).

La velocidad de descenso del $\mathrm{pH}$ se realiza de una manera gradual y más rápida, durante las 12 primeras horas post mortem, para luego casi estabilizarse hacia las 24 horas. Se reporta que cuando el valor del $\mathrm{pH}$ es menor a 6.0 durante la primera hora post mortem y la temperatura de la carne está próxima a $35^{\circ} \mathrm{C}$, se estaría frente a una carne PSE (pale, soft, exudative), que tiene una coloración pálida con intensa exudación, la cual es una anomalía común en ciertos cerdos (Culau, 1991). De otro lado, también se reporta que valores de $\mathrm{pH}$ por encima de 6.0 a las 24 horas post mortem, denotan una carne DFD (dark, firm, dry), la cual se caracteriza por una elevada retención de agua y una coloración oscura (Apple et al., 1995; Tarrant y Sherinton, 1980). Estos dos tipos o aspectos de la carne son indeseables por el consumidor, porque sus propiedades sensoriales son desagradables (Pearson, 1994). Por otro lado, los valores del $\mathrm{pH}$ de la carne a las 24 horas post mortem, pueden sufrir alteraciones debidas al uso de drogas o a condiciones de stress pre sacrificio a las que son sometidos los animales (Sanz et al., 1996).

El primer trabajo en el Perú sobre la determinación de la "acidez actual" (pH) de las carnes de vacuno lo realizó Izaguirre (1948) mediante el método colorimétrico, encontrando valores de $\mathrm{pH}$ de $6.3-6.5$ a las 4 horas post mortem. Estos valores son mayores a los medidos en el presente estudio, donde se encontró un valor de $\mathrm{pH}$ de 6.14 para las 4 primeras horas en animales Holstein y de 6.05 en Nelore. Estas diferencias podrían deberse a la precisión de los instrumentos usados, ya que las cintas colorimétricas no tienen la precisión del potenciómetro utilizado en el presente estudio y que además corrige los valores en base a la temperatura. Resultados más recientes en carcasas sin estimulación eléctrica de animales Holstein y Brahman (Mendoza, 2003) indican valores de $\mathrm{pH}$ ligeramente más elevados a los hallados en el presente trabajo a las 24 horas post mortem; sin embargo, señalaron valores de $\mathrm{pH}$ cercanos a 6.0 a las 2 horas post mortem. Estas diferencias podrían atribuirse a que los animales de aquel estudio no estuvieron lo suficientemente descansados, que sufrieron algún estrés, que el proceso de beneficio no fue apropiado o que no se empleó el factor de corrección con respecto a la temperatura durante la medición del pH.

\section{Conclusiones}

- Los valores de pH cárnico fueron mayores en toros Holstein que en toros Nelore $(\mathrm{p}<0.05)$.

- La variación de los valores de pH cárnico en el músculo Longissimus dorsi de toros Holstein y Nelore, siguen una tendencia similar durante las primeras 24 horas post mortem, mostrando un rápido descenso durante las primeras 12 horas.

- El pH de la carne a las 24 horas post mortem fue de 5.57 para el Holstein y de 5.40 para el Nelore, indicando una buena acidez para inhibir el posible crecimiento microbiano y garantizar una maduración apropiada.

\section{Limbratura Citada}

1. Alves, A. 1980. El cebú. Uteha. México. $44 \mathrm{p}$.

2. Apple, J.K.; M.E. Dikeman; J.E. Minton; R.M. McMurphy; M.R. Fedde; D.E. Leight; J.A. Unruh. 1995. Effects of restrain and isolation stress an epidural blockade on endocrine and blood metabolite status, muscle glycogen metabolism, and incidense of dark-cutting longissimus muscle of sheep. J. Anim. Sci. 73: 2295-2307.

3. Culau, P. 1991. Efeito da distancia abetedouro e temperatura de descanso pre-abate sobre a qualidade da carne da suína. Dissertaçâo-mestrado em Zootec- 
nia. Universidade Federal do Rio Grande do Sul. Porto Alegre. 132 p.

4. Devine, C.E.; B.B. Chystal; C.L. Devey: 1983. Effects of nutrition in lambs and subsequent postmortem biochemical changes in muscle. N.Z.J. Agric. Res. 26: 53-57.

5. Effenberger, G; K. Shotte. 1972. Empaquetado de la carne y productos cárnicos.p 51. Ed. Acribia. Zaragoza, España.

6. Fehlhaber, K.; P. Janetschke. 1995. Higiene veterinaria de alimentos. p 229233. Ed. Acribia. Zaragoza, España.

7. Izaguirre, A. 1948. Determinación de la acidez actual de las carnes. Tesis de Bachillerato. Facultad de Medicina Veterinaria, Univ. Nacional Mayor de San Marcos. Lima. $42 \mathrm{p}$.

8. Flores, A. 1997. Aplicación de los sistemas en engorde de vacunos. En: Engorde intensivos de vacunos. Programa de Invesigación y Proyección Social en Carnes. Universidad Nacional Agraria La Molina. Lima. 7 p.

9. Mendoza, F. 2003. Efecto de la estimulación eléctrica en el $\mathrm{pH}$ de bovino. Tesis de Bachillerato. Facultad de Zootecnia, Univ. Nacional Agraria La Molina. Lima. 78 p.

10. Murray, R.; P. Mayes; D. Granner; V. Rodwell. 2001. Bioquímica de Harper. $15^{\mathrm{a}}$ ed. Manual Moderno. México D.F.
II. Pardi, M.C.; I.F Santos; E.R. Souza; H.S. Pardi. 1993. Ciência, higiene e tecnologia da carne: Tecnología da sua obtençâo e transformaçao. p 586. Vol. 1. Centro Editorial e Grafico da Universidade da Goiás.

12. Pearson, A.M. 1994. La función muscular y los cambios post mortem. En: Price, J. F.; B.S Schweigent. The Science of Meat Products. $3^{\text {ra }}$ ed. p 139-174. Ed. Acribia. España.

13. Prändl, O.; A. Fisher; T. Schmidhofer; H. Sinell. 1994. Tecnología e higiene de la carne. Ed. Acribia. Zaragoza. 757 p.

14. Sánchez. G 1999. Ciencia básica de la carne. p 229-233. Fondo Nacional Universitario. Santafé de Bogotá.

15. Sanz, M.C.; M.T. Verde; T. Sáez; C. Sañudo. 1996. Effect of bread on the muscle glycogen content and dark cutting incident in stressed young bulls. Meat Sci. 43: 37-42.

16. Schackelford, D.; T. Wheeler; M. Koohmaraie. 1995. Relationship between shear force and trained sensory panel tenderness ratings of 10 major muscles from Bos indicus and Bos taurus cattle. J. Anim. Sci. 73: 33333340.

17 Tarrant, P.V.; J. Sherinton. 1980. An investigation of ultimate $\mathrm{pH}$ in the muscles of commercial beef carcasses. Meat Sci. 4: $287-292$. 